\title{
RELATIVE LIE ALGEBRA COHOMOLOGY REVISITED
}

\author{
MARK MUZERE
}

(Communicated by Maurice Auslander)

\begin{abstract}
In this paper it is shown that relative Lie algebra cohomology is related to relative cohomology for restricted Lie algebras by a spectral sequence. Also an interpretation of the relative cohomology groups for Lie algebras in terms of the relative right derived functors of a certain relative derivative functor is given.
\end{abstract}

\section{INTRODUCTION}

Throughout this paper let $(g, f)$ denote a pair of finite dimensional Lie algebras over a field $k$, where $f$ is a subalgebra of $g$, and let $(U g, U f)$ denote the corresponding pair of universal enveloping algebras of $g$ and $f$. For a $g$-module $W$, let $H^{n}(U g, U f, W)$ denote the relative cohomology groups defined by $H^{n}(U g, U f, W)=\operatorname{Ext}_{U g, U f}^{n}(k, W)$, where $\operatorname{Ext}_{U g, U f}^{n}(k, W)=$ $H^{n}\left(\operatorname{Hom}_{U g}(X ., W)\right)$ and $X . \rightarrow k \rightarrow 0$ is a $(U g, U f)$-projective resolution of $k[\mathrm{H}]$.

In this paper we continue our study of the relative cohomology groups $H^{n}(U g, U f, W)$ started in [M]. (Also see [B-W, H] for related works.)

First if $(g, f)$ is a pair of restricted Lie algebras, let $(V g, V f)$ denote their restricted enveloping algebras (cf. $\S 1$ ). The reader is referred to [J] for more details. Define relative cohomology groups $H^{n}(V g, V f, W)$ for the pair of restricted Lie algebras $(g, f)$ by $H^{n}(V g, V f, W)=\operatorname{Ext}_{V g, V f}^{n}(k, W)$, where $W$ is a restricted $g$-module. More precisely,

$$
\operatorname{Ext}_{V g, V f}^{n}(k, W)=H^{n}\left(\operatorname{Hom}_{V g}(X ., W)\right),
$$

where $X . \rightarrow k \rightarrow 0$ is a $(V g, V f)$-projective resolution of $k$. The reader may consult $[\mathrm{H}]$ for further details. It is shown here that the relative cohomology groups $H^{n}(U g, U f, W)$ and $H^{n}(V g, V f, W)$ are related by the following spectral sequence.

$$
\text { Let } 0 \rightarrow W \rightarrow I^{\circ} \text { be a }(U g, U f) \text {-injective resolution of } W \text {. }
$$

Then there is a first quadrant spectral sequence

Received by the editors April 18, 1989 and, in revised form, May 31, 1989.

1980 Mathematics Subject Classification (1985 Revision). Primary 17B56; Secondary 18G25. 


$$
E_{2}^{i, j}=H^{i}\left(V g, V f, H^{j}\left(\operatorname{Hom}_{0(g)}\left(k, I^{\cdot}\right)\right)\right) \Rightarrow H^{i+j}(U g, U f, W),
$$

where $0(g)$ is as defined at the beginning of $\S 2$.

Now assume $(g, f)$ to be a pair of not necessarily restricted Lie algebras. Let $\widehat{H}^{n}(g, f, W)$ denote the relative right derived functors of a certain relative derivation functor (cf. $\S 1)$. In [M], the group $H^{1}(U g, U f, W)$ has been identified with certain relative derivations modulo the inner ones. Here we provide a new interpretation of the higher relative cohomology groups $H^{n}(U g, U f, W)$. Namely,

$$
H^{n+1}(U g, U f, W) \cong \widehat{H}^{n}(g, f, W) \text { for } n \geq 1 .
$$

In $\S 2$, we construct the following Hochschild-Serre type spectral sequence.

(2.1) Let $f<h<g$ be a tower of Lie algebras where $h$ is an ideal of $g$, $\bar{g}=g / h$, and let $0 \rightarrow W \rightarrow I^{*}$ be a $(U g, U f)$-injective resolution of $W$. Then there is a first quadrant spectral sequence

$$
E_{2}^{i, j}=H^{i}\left(U \bar{g}, H^{j}\left(\operatorname{Hom}_{U h}\left(k, I^{*}\right)\right)\right) \Rightarrow H^{i+j}(U g, U f, W) .
$$

Moreover, if $f=0$, we obtain the Hochschild-Serre spectral sequence

$$
H^{i}\left(U \bar{g}, H^{j}(U h, W)\right) \Rightarrow H^{i+j}(U g, W) .
$$

Result (2.1) highlights certain subtleties in relative cohomology. The main observation here is that computation of the relative cohomology groups $H^{n}(U g, U f, W)$ is quite involved and in most cases a hopeless undertaking. These ideas will be made more precise in the appendix which deals with the analogous theory of relative group cohomology. An interpretation of the $E_{2}$ term of (3.1), a group cohomology analogue of (2.1) in terms of some familiar gadgets $[\mathrm{K}]$, turns out to be false.

\section{COMPARISON THEOREMS}

Unless stated otherwise throughout this section $(g, f)$ will be a pair of restricted Lie algebras, and $W$ will denote a restricted $g$-module. Define the restricted eveloping algebra of $g$ by $V g=U g \otimes_{0(g)} k$, where $0(g)$ is the central subalgebra of $U g$ generated by $x^{p}-x^{[p]}, x \in g$. Here $p$ is the characteristic of $k$. Let $V f$ be defined similarly. It is easy to see that there is a map $\varphi: H^{*}(V g, V f, W) \rightarrow H^{*}(U g, U f, W)$. Later we shall show that $H^{1}(V g, V f, W)$ is a subgroup of $H^{1}(U g, U f, W)$. First we show that the relative cohomology groups $H^{n}(V g, V f, W)$ and $H^{n}(U g, U f, W)$ are related by a spectral sequence. In fact, this is achieved by a generalization of [F-P, Proposition 5.3].

1.1. Theorem. Let $0 \rightarrow W \rightarrow I^{*}$ be a $(U g, U f)$-injective resolution of $W$. Then there is a first quadrant spectral sequence

$$
E_{2}^{i, j}=H^{i}\left(V g, V f, H^{j}\left(\operatorname{Hom}_{0(g)}\left(k, I^{\cdot}\right)\right)\right) \Rightarrow H^{i+j}(U g, U f, W) .
$$


Proof. That $\operatorname{Hom}_{0(g)}\left(k, I^{*}\right)$ is a complex of $V g$-modules is clear. Let $J . \rightarrow$ $k \rightarrow 0$ be a $(V g, V f)$-projective resolution of $k$. Now form the double complex $X_{i j}=\operatorname{Hom}_{V g}\left(J_{i}, \operatorname{Hom}_{0(g)}\left(k, I^{j}\right)\right)$ where $i, j \geq 0$. Following CartanEilenberg [C-E] define $I_{2}=H_{I} H_{I I}(X)$ and $I I_{2}=H_{I I} H_{I}(X)$. Thus

$$
I I_{2}^{i, j}= \begin{cases}H^{j}(U g, U f, W), & i=0 \\ 0, & i>0\end{cases}
$$

and $I_{2}^{i, j}=H^{i}\left(V g, V f, H^{j}\left(\operatorname{Hom}_{0(g)}\left(k, I^{*}\right)\right)\right)$. Therefore

$$
E_{2}^{i, j}=H^{i}\left(V g, V f, H^{j}\left(\operatorname{Hom}_{0(g)}\left(k, I^{\cdot}\right)\right)\right) \Rightarrow H^{i+j}(U g, U f, W) .
$$

1.2. Corollary. $H^{1}(V g, V f, W)$ is a subgroup of $H^{1}(U g, U f, W)$.

Proof. Appeal to the standard five-term exact sequence $0 \rightarrow E_{2}^{1,0} \rightarrow H^{1} \rightarrow \cdots$ associated to (1.1)

Recall the notation $W^{f}=\{w \in W \mid f . w=0\}$. If we set $f=0$ in the following result, we obtain [F-P, Proposition 5.3].

1.3. Corollary. If $f$ is an ideal of $g, \bar{g}=g / f$, we have

$$
E_{2}^{i, j}=H^{i}\left(V \bar{g}, H^{j}\left(0(\bar{g}), W^{f}\right)\right) \Rightarrow H^{i+j}\left(U \bar{g}, W^{f}\right) .
$$

Proof. This follows from (1.1) after making the following identifications. If we analyse the standard $(V g, V f)$-projective resolution of $k$, we obtain $H^{*}(V g, V f, W) \cong H^{*}\left(V \bar{g}, W^{f}\right)$. Since $H^{*}(U g, U f, W) \cong H^{*}\left(U \bar{g}, W^{f}\right)$, we may take the $(U g, U f)$-injective resolution of $W$ in (1.1) to be an actual $U \bar{g}$-injective resolution of $W^{f}$. Therefore

$$
H^{*}\left(\operatorname{Hom}_{0(g)}\left(k, I^{*}\right)\right) \cong H^{*}\left(0(\bar{g}), W^{f}\right) .
$$

In what follows let $(g, f)$ be a pair of not necessarily restricted Lie algebras. We now show that the higher relative cohomology groups $H^{n}(U g, U f, W)$ may be identified with the relative right derived functors of a relative derivation functor defined below. Recall that a linear map $h: g \rightarrow W$ such that $h([x, z])=x \cdot h(z)-z \cdot h(x)$ for all $x, z \in g$ is called a derivation. Denote the set of all derivations from $g$ to $W$ that vanish on $f$ by $\operatorname{Der}(g, f, W)$. If $f$ is an ideal of $g, \bar{g}=g / f$, then $\operatorname{Der}(g, f,) \cong \operatorname{Der}(\bar{g}$,$) . \operatorname{Der}(g, f$,$) is clearly$ a covariant left-exact functor from $g$-modules to $k$-vector spaces. Therefore it gives rise to certain relative Ext functors. These functors generalize certain well-known results in ordinary Lie algebra cohomology.

The following lemma is the key to the identification proposed above.

1.4. Lemma (Muzere [M]). Let I $g=\operatorname{ker}(\varepsilon: U g \rightarrow k)$ and let If be defined similarly. Then, for any $g$-module $W$, the linear map

$$
\eta(\nu): \operatorname{Hom}_{U g}(I g / U g I f, W) \rightarrow \operatorname{Der}(g, f, W)
$$


defined by

$$
\eta(\nu)(h)=h \circ \nu \circ i
$$

where $i: g \rightarrow U g$ is the canonical embedding and $h \circ \nu$ is the extension of

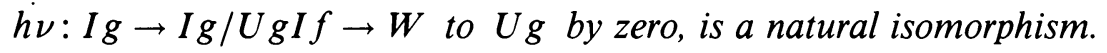

Let $\hat{H}^{n}(g, f, W)$ denote the $n$th relative right derived functor of $\operatorname{Der}(g, f$,$) with coefficients in W$. By Lemma 1.4,

$$
\widehat{H}^{n}(g, f, W) \cong \operatorname{Ext}_{U g, U f}^{n}(I g / U g I f, W)
$$

for $n \geq 0$. Consequently $\widehat{H}^{n}(g, f, W)$ can be manipulated by using standard techniques in homological algebra. For example, since there are enough $(U g, U f)$-injectives, $\widehat{H}^{n}(g, f$,$) is coeffaceable for n \geq 1$. In view of this, it is an easy matter to prove the following comparison theorem.

1.5. Theorem. $H^{n+1}(U g, U f, W) \cong \widehat{H}^{n}(g, f, W)$ for $n \geq 1$.

Proof. Since $I g / U g I f \cong \operatorname{ker}\left(U g \otimes_{U f} k \rightarrow k\right)$, we have

$$
H^{n+1}(U g, U f, W) \cong \operatorname{Ext}_{U g, U f}^{n}(I g / U g I f, W) \quad \text { for } n \geq 1 .
$$

If $f=0$, we obtain the following result [Ba-R].

1.6. Corollary. $H^{n+1}(U g, W) \cong \widehat{H}^{n}(g, W)$ for $n \geq 1$.

\section{HOCHSCHILD-SERRE TYPE SPECTRAL SEQUENCE}

In this section $(g, f)$ will be a pair of not necessarily restricted Lie algebras and $W$ will denote a $g$-module. In [M], it was pointed out that computations in relative cohomology seem to be very involved. The following result reinforces this point of view. $\S 3$ contains further elaboration on these matters.

2.1. Theorem. Let $f<h<g$ be a tower of Lie algebras where $h$ is an ideal of $g, \bar{g}=g / h$, and let $0 \rightarrow W \rightarrow I^{*}$ be a $(U g, U f)$-injective resolution of $W$. Then there is a first quadrant spectral sequence

$$
E_{2}^{i, j}=H^{i}\left(U \bar{g}, H^{j}\left(\operatorname{Hom}_{U h}\left(k, I^{\cdot}\right)\right)\right) \Rightarrow H^{i+j}(U g, U f, W) .
$$

Moreover, if $f=0$, we obtain the Hochschild-Serre spectral sequence

$$
H^{i}\left(U \bar{g}, H^{j}(U h, W)\right) \Rightarrow H^{i+j}(U g, W) .
$$

Proof. Let $J$. $\rightarrow k \rightarrow 0$ be a $U \bar{g}$-projective resolution of $k$. Next form the double complex $X_{i j}=\operatorname{Hom}_{U \bar{g}}\left(J_{i}, \operatorname{Hom}_{U h}\left(k, I^{j}\right)\right)$, where $i, j \geq 0$, and proceed as in the proof of (1.1).

In the following result we extend the well known exact sequence in ordinary Lie algebra cohomology to the relative case. 
2.2. Corollary. Let $f<h<g$ be as in (2.1). Then there is an exact sequence

$$
\begin{aligned}
0 \rightarrow H^{1}(U g, U h, W) \rightarrow H^{1}(U g, U f, W) \rightarrow E_{2}^{0,1} & \\
& \rightarrow H^{2}(U g, U h, W) \rightarrow H^{2}(U g, U f, W) .
\end{aligned}
$$

Proof. This follows from the standard five-term exact sequence associated to (2.1) and the fact that $H^{n}(U g, U h, W) \cong H^{n}\left(U \bar{g}, W^{h}\right)$ for $n \geq 0$.

We next show that under certain conditions the term $E_{2}^{0,1}$ has a nice interpretation.

2.3. Corollary. Let the characteristic of $k$ be 0 and let $f<h<g$ be as in (2.1) with $h$ the radical of $g$. Then if $W$ is finite dimensional, $E_{2}^{0,1} \cong$ $H^{1}(U g, U f, W)$.

Proof. Appeal to (2.2) and the two well-known Whitehead lemmas.

\section{APPENDIX}

Throughout this section $G$ will denote a finite group. Let $(G, H)$ be a pair of groups where $H$ is a subgroup of $G$. For a (left) $G$-module $A$ let $H^{n}(G, H, A)$ denote the relative cohomology groups defined by $H^{n}(G, H, A)=$ $H^{n}\left(\operatorname{Hom}_{G}(X ., A)\right)$, where $X . \rightarrow Z \rightarrow 0$ is a $(G, H)$-projective resolution of $Z$ [H].

In group cohomology, the cohomology groups of $G$ are especially tractable if $G$ has periodic cohomology [C-E] or if it admits a Hochschild-Serre spectral sequence argument. Extensions of these techniques to the relative case can be found in [Har], $([\mathrm{K}],[\mathrm{S}])$ respectively. In this section we shall be concerned with the latter. In fact, we have

3.1. Theorem (Katamadze [K]). If $H<K<G$ is a tower of groups where $K$ is normal in $G, \bar{G}=G / K$, and if $0 \rightarrow A \rightarrow I^{*}$ is a $(G, H)$-injective resolution of $A$, there is a first quadrant spectral sequence

$$
E_{2}^{i, j}=H^{i}\left(\bar{G}, H^{j}\left(\operatorname{Hom}_{K}\left(Z, I^{\cdot}\right)\right)\right) \Rightarrow H^{i+j}(G, H, A) .
$$

Proof. Let $J . \rightarrow Z \rightarrow 0$ be a $\bar{G}$-projective resolution of $Z$. The result now follows from a standard argument applied to the double complex $X_{i j}=$ $\operatorname{Hom}_{\bar{G}}\left(J_{i}, \operatorname{Hom}_{K}\left(Z, I^{j}\right)\right)$, where $i, j \geq 0$.

This spectral sequence would facilitate computations in relative group cohomology if determination of the groups $H^{j}\left(\operatorname{Hom}_{K}\left(Z, I^{*}\right)\right)$ was plausible. We now show that there is a persistent difficulty in determining these groups. More precisely, we have

3.2. Lemma. The interpretation $H^{j}\left(\operatorname{Hom}_{K}\left(Z, I^{*}\right)\right)=H^{j}(K, H, A)$ for $j \geq 0$ in $[\mathrm{K}]$ is false in general.

Proof. To see this we produce a contradiction to the following well-known result. 
3.3. Theorem (Blowers [B1]). Let $k=F_{p}$ and $G$ be the Sylow p-subgroup of the symmetric group on $p^{t}$ elements, where $t \geq 2$, and let $H$ be some appropriate nonnormal subgroup of $G$. Then the cohomology ring $H^{*}(G, H, k)$ is not finitely generated.

Assume the interpretation given in (3.2) of the $E_{2}$-term of (3.1). Let $k$ be as in (3.3), and let $G$ be any $p$-group with a nonnormal subgroup $H$. By a standard fact in group theory there is a tower of subgroups $H_{1}=H<$ $H_{2}<\cdots<H_{r-1}<H_{r}=G$ where, for each $i, H_{i}$ is normal in $H_{i+1}$ and $H_{i+1} / H_{i} \cong C_{p}$, a cyclic group of order $p$. If we take $K=H_{i}$ for appropriate $i,(3.1)$ yields spectral sequences

$$
E_{2}^{s, t}=H^{s}\left(C_{p}, H^{t}\left(H_{i}, H, k\right)\right) \Rightarrow H^{s+t}\left(H_{i+1}, H, k\right),
$$

where $2 \leq i \leq r-1$. Thus

Observe that if $i=2$, we have our initial tower of groups $\mathrm{H}<\mathrm{H}_{2}<\mathrm{H}_{3}$.

$$
\begin{aligned}
E_{2}^{s, t} & =H^{s}\left(C_{p}, H^{t}\left(H_{2}, H, k\right)\right) \\
& \cong H^{s}\left(C_{p}, H^{t}\left(C_{p}, k\right)\right) \quad(\text { by }[\mathrm{A}, \text { Theorem 3.2] }) .
\end{aligned}
$$

Hence $H^{*}\left(H_{3}, H, k\right)$ is a finitely generated ring. Employing ideas of Evens [E] (see also [Be]) in an inductive argument, one obtains $H^{*}(G, H, k)$, a finitely generated ring. Now if we choose $G$ and $H$ as in (3.3), we obtain a contradiction as desired.

3.5. Lemma. If we take the ring of coefficients to be the rational integers $Z$, (3.2) also holds.

Proof. Take $G=D_{8}=g p\left\langle b, s \mid b^{2}=e=s^{4}, b^{-1} s b=s^{-1}\right\rangle, K=\left\{e, s^{2}, s b\right.$, $\left.s^{3} b\right\}$, and $H=\{e, s b\}$. Then we obtain a tower of groups $H<K<G$ where $K$ is normal in $G$. Observe that $H$ is not normal in $G$. Since $H$ is cyclic, we have an exact sequence

$$
0 \rightarrow H^{2}(G, H, Z) \rightarrow H^{2}(G, Z) \rightarrow H^{2}(H, Z)
$$

by [A, Theorem 7.3]. From Evens and Priddy [E-Pr] (see also [C-E, p. 250]) we get $H^{2}(G, Z) \cong Z_{2}^{2}$ and $H^{2}(G, H, Z) \cong Z_{2}$. Now the interpretation in (3.2) would yield $H^{2}(G, H, Z) \cong Z_{2}^{2}$ which is not consistent with the above calculation.

3.6. Remark. The argument in the proof of (3.5) also carries over to the tower of groups $H<K<G$, where $G=A_{4}, K=\{e,(12)(34),(13)(24),(14)(23)\}$ and $H=\{e,(12)(34)\}$.

\section{ACKNOWLEDGMENT}

It is a pleasure to thank Ed Cline and the referee for useful comments and suggestions. 


\section{REFERENCES}

[A] I. T. Adamson, Cohomology for nonnormal subgroups, Proc. Glasgow Math. Assoc. 2 (1954-56), 66-76.

[Ba-R] B. Barr and G. S. Rinehart, Cohomology as the derived functor of derivations, Trans. Amer. Math. Soc. 122 (1966), 416-426.

[Be] F. R. Beyl, The spectral sequence of a group extension, Bull. Sci. Math. 2nd edition Série 105 (1981), 417-434.

[Bl] J. Blowers, The cohomology rings of certain permutation representations, Proc. Amer. Math. Soc. 45 (1974), 157-163.

[B-W] A. Borel and N. Wallach, Continuous cohomology, discrete subgroups and representations of reductive groups, Annals of Math. Studies 94 (1980), Princeton University Press, Princeton, NJ.

[C-E] H. Cartan and S. Eilenberg, Homological Algebra, Princeton University Press, Princeton, NJ, 1956.

[E] L. Evens, The Cohomology of a finite group, Trans. Amer. Math. Soc. 101 (1961), 224-239.

[E-Pr] L. Evens and S. Priddy, The cohomology of the semidihedral group, Contemp. Mathematics 37 (1985), 61-72.

[Har] M. Hartis, Cupproduct, duality and periodicity for generalized group cohomology, Fund. Math. 66, 99-121.

[H] G. Hochschild, Relative homological algebra, Trans. Amer. Math. Soc. 82 (1956), 246-269.

[J] N. Jacobson, Lie algebras, Dover Publications, New York, 1962.

[K] R. Katamadze, On exact sequences for the relative Hoschschild cohomology, 104, Soobsch. Akad. Nauk, Gruzin SSR, 1981, pp. 21-23 (Russian).

[M] M. Muzere, Relative Lie algebra cohomology, J. Pure and Applied Algebra, 58 (1989,), 77-91.

[S] E. Snapper, Cohomology of permutations I. Spectral sequences, J. of Math. and Mech. 13 (1964), 133-161.

Department of Mathematics, Clark University, 950 Main Street, Worcester, MasSACHUSETTS 01610-1477 